\title{
Lobaplatin or Cisplatin Plus Etoposide for Treating Extensive Stage Small Cell Lung Cancer
}

\author{
Xin Zhou, Hongge Zhu, Yiyi Zhan and Chunling Liu* \\ Department of Lung Cancer, Tumor Hospital, Xinjiang Medical University, China
}

\begin{abstract}
Objective: To observe the short-term therapeutic effect, adverse reactions and hospitalization conditions of lobaplatin plus etoposide (EL) and cisplatin plus etoposide (EP) for advanced small cell lung cancer (SCLC).

Methods: A total of 46 patients with advanced SCLC were randomized into EL ( 23 patients) and EP ( 23 patients) groups. The two groups were treated for two cycles of 21 days. Short-term therapeutic effects, adverse reactions and hospitalization conditions were evaluated for the two groups after two cycles.

Results: The EL and EP groups did not differ substantial in objective response rate, hospital stay, or rate of leukocyte and platelet decrease. In the two groups, NSE (neuron-specific enolization enzyme) was reduced significantly after chemotherapy, but CEA (carcinoembryonic antigen) and CYFRA21 (cytokeratin 19 fragment) were not different between the two groups. In the EL group, the incidence of gastrointestinal reaction was reduced significantly and hospitalization cost was significantly increased.
\end{abstract}

Conclusion: EL and EP for the same period of SCLC is safe and effective chemotherapy regimens, EL group medical expenses increased significantly, but the digestive tract reaction decreased significantly.

\section{Keywords}

Cisplatin, Etoposide, Lobaplatin, Small cell-lung cancer

Primary bronchial lung cancer is a high morbidity and mortality tumor. Small cell lung cancer (SCLC), a kind of lung cancer, is about $15 \%$ to $20 \%$ of the total number of lung cancers [1]. The clinical characteristics are rapid increase, cell differentiation, and high degree of malignancy, with metastasis appearing early and widely. An extensive SCLC account for about $70 \%$ of the total number of SCLCs. Chemotherapy is the main method of treatment for extensive periods of SCLC. The etoposide combined with cisplatin (EP) scheme is standard for first-line chemotherapy of SCLC [2]. LBP (lobaplatin) is a new generation of platinum anticancer drugs. DDP (Cisplatin), which has a tumor suppression function, is similar to LBP with fewer adverse reactions [3]. We report diagnosis and treatment of 46 patients with extensive SCLC in a prospective, open-label case-control study.

\section{Materials and Methods}

Participants were chosen from patients from 1 June 2012 to 31 December 2015 at Xinjiang Tumor Hospital Pulmonary Medicine. Patients had extensive SCLC, 46 with initial diagnosis by cytology and/or pathological histology with an expected lifetime $>3$ months. Systemic assessment was routine blood, urine, and stool tests, liver and kidney function, electrolytes, blood coagulation series, electrocardiogram, chest CT (Computed Tomography), MRI (cranial magnetic resonance imag- ing), bone ECT (Emission Computed Tomography), neck or abdominal ultrasound systemic PET - CT (position-emission tomography Computed Tomography) check, diagnosis phase of extensive SCLC (using American Veterans Association lung cancer staging), chest CT to evaluate lesions, and PS score not in accord with chemotherapy indications and contraindications. Exclusion criteria were: (1) Other serious disease or metabolic autoimmune disease; (2) Severe functional disorders such as heart, liver, or kidney failure or fungal infections, severe bleeding tendency, granulocytopenia, or thrombocytopenia.

In accordance with the requirements of ethical research, explanations were given to patients or their authorized representative for all illnesses. Patients or their authorized person signed a consent agreement for chemotherapy. According

*Corresponding author: Chunling Liu, Department of Lung Cancer, Tumor Hospital, Xinjiang Medical University, China

Accepted: June 11, 2019

Published online: June 13, 2019

Citation: Zhou X, Zhu H, Zhan Y, et al. (2019) Lobaplatin or Cisplatin Plus Etoposide for Treating Extensive Stage Small Cell Lung Cancer. Ann Lung Cancer 3(1):60-63 
to confirmed order of entry into the group, 46 patients were randomized to an EP (joint cisplatin), or EI (joint Lobaplatin) group. Of 23 patients, 15 were men and 8 were women. Ages were 36-77 years with an average age of 45.7 and 46.1 ). In the El group of 23 patients, 16 were men and 7 were women. Ages were $35-78$ years with an average age of 47.3 and 65.8). No significant differences were seen in, for example, KPS scale (karnofsky performance status scale), gender, or age $(P>0.05)$.

\section{Treatment and medications}

(1) In the EP group, EP chemotherapy was DDP (Shandong Qilu Pharmaceutical Co.LTD in China), $30 \mathrm{mg} / \mathrm{m}^{2}$ in $250 \mathrm{ml} \mathrm{sa-}$ line static ,drops ,d1-3, and VP-16 (Jiangsu Hengrui Pharmaceutical Company in China) $80 \mathrm{mg} / \mathrm{m}^{2}$ in $250 \mathrm{ml}$ saline, drops, dl-d5. (2) In the El group, chemotherapy used El, which was LBP (Hainan Chang'an International Pharmaceutical Company Products in China) $30 \mathrm{mg} / \mathrm{m} \mathrm{5 \%}$ pt glucose injection $250 \mathrm{ml}$, static drops, d1, and VP- $1680 \mathrm{mg} / \mathrm{m}^{2}$ with physiological saline to $250 \mathrm{ml}$, static, d1-5. Both groups received 2 cycles of chemotherapy and chemotherapy for antiacid and antinausea treatment. Solutions were given at 21 days for one cycle. If bone marrow suppression, leukocyte, platelet inhibition to IV degree, need to adjust the dose of start next cycle, reduced by $25 \%$.

\section{Evaluation}

Standard patients who had an empty stomach in the morning had routine blood checks twice per week, and liver kidney function and electrolytes and ECG (electrocardiogram) once per week. Before and after two cycles of chemotherapy, blood was taken for lung cancer tumor-related antigen examination, to observe clinical adverse reactions, and for chest CT evaluation of lung lesion size changes. In accordance with the $\mathrm{NCl}$ - CTC - 3 ( $\mathrm{NCl}$ Dividing Standard Version. 3) Criterion for judging adverse reactions, divided as 0-IV, one period after chemotherapy to evaluate adverse reactions. For patients with severe adverse reactions, timely adjustment of dosage or termination of chemotherapy occurred.

Curative effect was evaluated by standard of curative effect evaluation in accordance with Solid Tumors Version 1.1 in solid tumors evaluation. Effects were divided into CR (com-

Table 1: Comparison of short-term objective therapeutic effects between two groups.

\begin{tabular}{|l|l|l|l|l|l|}
\hline Category & CR & PR & SD & PD & ORR (\%) \\
\hline EPgroup & 3 & 11 & 7 & 2 & $14(60.9)$ \\
\hline Elgroup & 4 & 12 & 6 & 1 & $16(79.6)$ \\
\hline
\end{tabular}

plete remission or complete response), PR (partial response or partial response), SD (stable diseases) or PD (progression disease). Continuous administration of two periodic evaluations of curative effects for patients in remission (CR or PR) was 4 weeks after confirmed curative effect. Calculations were based on the total number of cases of CR and PR response rate $(R R)$.

Adverse reactions were blood system reduction (white cells or platelets) and incidence of adverse gastrointestinal reactions.

Lung cancer tumor-related antigen serum for the two groups of patients before and after chemotherapy carcinoembryonic antigen (CEA), neuron-specific enolization enzyme (NSE) and cytokeratin 19 fragment (CYFRA21-1). Normal reference values were: CEA 0-3.4 ng/ml, NSE 0-15.2 ng/ $\mathrm{ml}$, CYFRA21-1 0-3.3 ng/ml comparing the two groups for chemotherapy compliance, twice of the medical expenses of chemotherapy, and length of hospital stay.

\section{Statistical methods}

Methods used SPSS 17.0 statistical software to analysis. Count data such as percentages by $\mathrm{X}$-ray inspection were measured as $x \pm s$ and average using independent sample t-tests and paired t-tests with $\mathrm{P}<0.05$ for differences that were statistically significant.

\section{Results}

\section{Two recent objective and efficient chemothera- pies}

We compared two groups of 46 patients who completed two cycles of chemotherapy. For one patient with bone marrow suppression, we adjusted the VP-16 and LBP dose for 2 cycles by $20 \%$. The two treatment groups, by objective efficiency ( $C R+P R>/ C R+S D+P D(C R+P R) \%)$, showed no significant differences $\left(X^{2}=0.383, P>0.05\right.$, Table 1$)$.

\section{Comparison of adverse reactions for two evalu- ated groups of 46 patients}

Gastrointestinal reactions were seen in $87.0 \%$ (20) of the EP group and $60.9 \%$ (14) of the EL group with significant differences between the two groups $(P<0.05)$ to I-II degrees of reaction. The two groups of patients did not experience clear effects on the cardiovascular system, urinary system, or toxic effects of the nervous system; no chemotherapy deaths occurred (Table 2).

For bone marrow suppression, the EP group had $73.9 \%$ white blood cell and $65.2 \%$ platelet declines with I-II degree.

Table 2: Comparison of short-term toxic adverse reactions between the two groups.

\begin{tabular}{|c|c|c|c|c|c|c|c|c|c|c|c|c|}
\hline \multirow{2}{*}{ Toxicity reaction } & \multicolumn{6}{|c|}{ EP } & \multicolumn{6}{|c|}{ EL } \\
\hline & 0 & I & II & III & IV & incidence \% & 0 & I & II & III & IV & incidence \% \\
\hline White blood cells reduced & 6 & 10 & 5 & 2 & 0 & 73.9 & 4 & 9 & 6 & 3 & 1 & 82.6 \\
\hline Thrombocytopenia & 8 & 10 & 4 & 1 & 0 & 65.2 & 3 & 13 & 5 & 2 & 0 & 87 \\
\hline Nausea and vomiting & 3 & 9 & 7 & 4 & 0 & 87 & 9 & 5 & 7 & 2 & 0 & $60.9^{*}$ \\
\hline
\end{tabular}


Table 3: Comparison of tumor marker levels before and after lung cancer chemotherapy in two groups.

\begin{tabular}{|c|c|c|c|}
\hline Group & CEA & CYFRA21-1 & NSE \\
\hline \multicolumn{4}{|l|}{ EP } \\
\hline Before treatment & $3.97 \pm 1.29$ & $3.98 \pm 1.04$ & $61.68 \pm 16.20$ \\
\hline After treatment & $3.83 \pm 1.33$ & $3.87 \pm 1.06$ & $17.74 \pm 4.84^{*}$ \\
\hline \multicolumn{4}{|l|}{ EL } \\
\hline Before treatment & $3.80 \pm 0.95$ & $3.49 \pm 1.03$ & $67.44 \pm 12.21$ \\
\hline After treatment & $3.64 \pm 0.99$ & $3.37 \pm 0.87$ & $17.30 \pm 5.38^{*}$ \\
\hline
\end{tabular}

Compared with before treatment, ${ }^{*} \mathrm{P}<0.01$.

Table 4: Status of two groups in hospital characteristics.

\begin{tabular}{|l|l|l|}
\hline Factors & EP group & EL group \\
\hline $\begin{array}{l}\text { Chemotherapy } \\
\text { compliance (\%) }\end{array}$ & 91.3 & 95.7 \\
\hline $\begin{array}{l}\text { Length of hospital } \\
\text { stay (d) }\end{array}$ & $15.83 \pm 1.11$ & $15.65 \pm 1.26$ \\
\hline Medical fees (¥) & $9343.09 \pm 314.19$ & $13224.35 \pm 295.30^{*}$ \\
\hline
\end{tabular}

Compared with EP group, $* p<0.01$.

In the EL group, white blood cells decreased $82.6 \%$ and platelets $87.0 \%$, also with I-II degree. No significant difference was seen between the two groups $(P>0.05)$.

\section{Serum tumor markers in lung cancer patients in different chemotherapy regimens}

In the EP and EL chemotherapy groups, NSE was significantly lower than before treatment $(P<0.05)$. CEA did not change significantly before and after chemotherapy using a CYFRA21-1 test (Table 3).

\section{Comparing two groups for patient condition}

In the EL group, one patient at the third admission refused chemotherapy signed after refusing medical responsibility, enhanced nutrition and immune support treatment. Chemotherapy compliance in the two groups was good, with no significant differences between groups $(P>0.05)$. Comparing the EL and EP groups showed no decrease in hospitalization time of chemotherapy and no significant difference $(P>0.05)$; however, costs and expenses related to medical hospital chemotherapy increased significantly with significant differences between groups $(P<0.05)$ (Table 4$)$.

\section{Discussion}

Incidence and mortality of lung cancer were the highest in malignant tumors [4-5]. SCLC accounts for about $15 \%-20 \%$ of lung cancer incidence. New NCCN (National Comprehensive Cancer Network) guidelines point that few patients can have early surgical excision, but for most, the priority is a chemotherapy treatment plan to control tumor progression, prolong survival time, and improve the quality of life [6-8]. Tumor molecular biology research in recent years has expanded and detection of serum tumor markers of lung cancer has developed rapidly, especially for early lung cancer screening, which is widely used for efficacy determination and prognosis evaluation [8]. Lung cancer markers with comparatively wide clinical application and effects include CYFRA21-1, CEA, NSE, whose level can reflect the stage of clinical disease.

CEA is an antigen that can cause an immune response and exists widely in the origin of the germ layer within digestive system cancers and in the embryonic normal digestive tube in the organization, with traces in normal human serum $[9,10]$. CEA is a broad-spectrum tumor marker that can reflect the existence of a wide variety of tumors. It can be used to judge curative effects for colorectal, breast and lung cancer. For illness development, monitoring and prognosis estimation, it is one of the better tumor markers, but its specificity is not strong and sensitivity is not high. It can be used in the diagnosis of lung cancer, especially adenocarcinoma content, with tumor size and staging, metastasis and prognosis [10].

CYFRA21-1 is a cytokeratin 19 fragment. Its main composition in the cellular structure of two monoclonal antibodies Cytokeratin 19 mainly exists in, for example, lung and esophageal cancer in the cytoplasm of tumor cells of epithelial origin such as lung adenocarcinoma. Expression of lung squamous carcinoma CYFRA21-1, when cells are cancerous, is due to necrosis of tumor cells to release CYFRA21-1 content in the blood; this can reflect disease prognosis and treatment effects [11]. Product related to CK19 (Cytokeratin 19) are serum CYFRA21-1 for organ-specificity tumor markers. In clinical application, comprehensive analysis should be combined with clinical data for more accurate judgments [11].

NSE is specific to neurons and neuroendocrine cells. It is an acidic protease and neuroendocrine tumor-specific sign, such as for neuroblastoma, medullary thyroid carcinoma and SCLC (70\%). It can be used for differential diagnosis, condition monitoring, curative effect evaluation and prediction of recurrence. Presently, NSE is considered to be most specific and most sensitive to SCLC markers. In the literature [12], SCLC chemotherapy curative effects are associated with serum NSE. Dynamic monitoring revealed increased serum NSE in SCLC for the extensive stage greater than the Limited stage within a limited time. NSE declined indicates effective treatment. With tumor recurrence, hair and blood NSE increases again [12].

EP solutions are standard chemotherapy for SCLC [13]. Currently platinum drugs which are clinically used with cisplatin, nida cisplatin, carboplatin, oxaliplatin and others are used commonly. For a long time cisplatin treatment, most patients become resistant to cisplatin and disease recurs. Explanations include enhanced cell detoxification of cisplatin, inhibition of cell apoptosis, and enhancement of DNA repair ability. However, the renal toxicity of cisplatin and the ototoxicity and gastrointestinal reactions limit its clinical application. Although as an alternative to cisplatin, carboplatin EC solutions are recommended as standard in NCCN guidelines, the bone marrow inhibition reaction is larger. The role in the bureau of SCLC within a time period is not clear, especially in older patients with SCLC application risks [14]. Therefore, finding new platinum drugs is necessary.

LBP (lobaplatin) is from the Germany Amadeus company, 
which is pioneering research and development of third-generation platinum anticancer drugs. In 2005, the Chinese Food and Drug Administration approved Lobaplatin for listing in China for treatment of advanced breast cancer, chronic myelogenous leukemia and SCLC [14]. Lobaplatin is a generalized alkylating agent. Research shows that the drug has good stability and high solubility and is a wide spectrum antineoplastic with strong antitumor activity. It has fewer adverse reactions and and other advantages. The pharmacokinetics of Lobaplatin are quick effect, long duration, high concentration in tumor tissues and characteristics of low concentration in plasma. The drug has good selectivity. Its antitumor effect shows no cross resistance with cisplatin.

The mechanism of action of Lobaplatin is similar to cisplatin. It interferes with the tumor cell cycle mainly through the formation of Pt - GG and Pt. AG crosslink chains, blocking replication of DNA and transcription. It further causes DNA damage, affecting specific gene expression of tumor cells. The main adverse reactions are bone marrow suppression, especially thrombocytopenia, as a dose-limiting adverse reaction. Other common adverse reactions including gastrointestinal reaction (III degrees), renal toxicity and neurotoxicity are not obvious [15].

The results of this study showed that in the broad tissue stage of SCLC chemotherapy, compared with the classic EP scheme, an El program objectively and efficiently but not significantly reduced bone marrow toxicity with reductions in platelets and white blood cells reduce. The incidence was not significantly increased, but gastrointestinal reaction decreased significantly (common I-Il degrees). Two groups of serum tumor markers in lung cancer patients were significantly reduced after treatment compared to before treatment. However, no obvious change was seen in CEA and CYFRA21-1 before and after chemotherapy. In two groups of patients receiving chemotherapy for the third time with good treatment adherence, the El group had no decrease in hospital time, but chemotherapy treatment in hospital significantly increased. Significant differences were observed between the two groups. For extensive-stage SCLC, the EL scheme of chemotherapy had a curative effect with good reactions, especially gastrointestinal reactions such as nausea and vomiting, which were significantly reduced. Compliance was good, which has important clinical value, but can also lead to increased hospitalization and medical expenses.

The number of samples in this study was small. We had no long-term followup and need to continue to enlarge the sample size to observe curative effects of long-term followup. Thus, a more accurate and comprehensive evaluation of Lobaplatin is needed to determine its broad SCLC chemotherapy application value.

\section{References}

1. Kalemkerian GP, Akerley W, Bogner P, et al. (2012) Small cell lung cancer. J Natl Compr Canc Netw 11: 78-98.

2. Chalian H, Tore HG, Horowitz JM, et al. (2011) Radiologic assessment of response to therapy: Comparison of RECIST Versions 1.1 and 1.0. Radiographics 31: 2093-2105.

3. Guo Wanfeng, Liao Guoqing, Gao Hongjun, et al. (2013) Randomized comparison of lobaplatin plus etoposide and cisplatin plus etoposide chemotherapy in patients with extensive-stage small cell lung cancer. Chinese-German Clin Oncol 12: 365-368.

4. Li X, Asmitananda T, Gao L, et al. (2012) Biomarkers in the lung cancer diagnosis: A clinical perspective. Neoplasma 59: 500-507.

5. Xiao-qi Huang (2012) Serum ProGRP in elderly patients with advanced non-small-cell lung cancer, TPS, NSE detection. The Clinical Significance of the Chinese Journal of Gerontology 32: 2053-2054.

6. Jin Hur, Hye-Jeong Lee, Ji Eun $\mathrm{Na}$, et al. (2012) Additional diagnostic value of tumor markers in cytological fluid for diagnosis of non-small- cell lung cancer. BMC Cancer 12: 392.

7. Wagner IC, Guimarães MJ, da Silva LK, et al. (2007) Evaluation of serum and pleural levels of the tumor markers CEA, CYFRA21-1 and CA 15-3 in patients with pleural effusion. J Bras Pnuemol 33: 185-191.

8. Zhao Huan, Bi Gong Xia (2012) NSE and CYFRA21-1, three tumor markers CA125 joint detection value in the diagnosis of lung cancer. China Laboratory Diagnostics 2012: 1295-1296.

9. Chu XY, Hou XB, Song WA, et al. (2011) Diagnostic values of SCC, CEA, Cyfra2l-1 and NSE for lung cancer in patients with suspicious pulmonary masses: A single center analysis. Cancer Biol Ther 11: 995-1000.

10. Salgia R, Hensing T, Campbell N, et al. (2011) Personalized treatment of lung cancer. Semin Oncol 38: 274-283.

11. Meley D, Spiller DG, White MR, et al. (2010) p53 mediated delayed NF-rcB activity enhances etoposide induced cell death in medulloblastoma. Cell Death Dis 1: e41.

12. Califano R, Abidin AZ, Peck R, et al. (2012) Management of small cell lung cancer: Recent developments for optimal care. Drugs 72: 471-490.

13. Yang Liuqiu, Qin Shuwel (2009) The third generation of platinum drugs research advances of los platinum. Journal of Clinical Oncology 14: 1134-1139.

14. Monneret $C$ (2011) Platinum anticancer drugs. From serendipity to rational design. Ann Pharm Fr 69: 286-295.

15. Xie Cy, Xu Yp, Jin W, et al. (2012) Antitumor activity of lobaplatin alone or in combination with antitubulin agents in non-small-cell lung cancer. Anticancer Drugs 23: 698-705.

DOI: $10.36959 / 825 / 579$

Copyright: (C) 2019 Zhou X, et al. This is an open-access article distributed under the terms of the Creative Commons Attribution License, which permits unrestricted use, distribution, and reproduction in any medium, provided the original author and source are credited. 